\title{
3D Reconstruction of Neurons from Confocal Image Stacks and Visualization of Computational Modeling Experiments
}

\author{
Susanne Schönknecht ${ }^{1}$, Carsten Duch $^{2}$, Klaus Obermayer ${ }^{1}$, Michael Sibila $^{1}$ \\ ${ }^{1}$ Fachgebiet Neuronale Informationsverarbeitung, Fakultät IV, TU-Berlin \\ ${ }^{2}$ School of Life Sciences, Arizona State University, USA \\ susanne@cs.tu-berlin.de
}

\begin{abstract}
In this study we perform precise geometrical 3D reconstructions of high complex neuronal architectures. First, confocal microscopy was used to scan neurons with submicron resolution. Second, we extracted the center-lines and diameters of the neuron by means of our reconstruction method, and third we used these metric data to generate compartment models that were transported into the proprietary format of modeling software such as NEURON or GENESIS. Fourth, routines were developed in the scripting language of the respective modeling program to perform computational modeling, and finally we transferred the modeling results to the visualization program AMIRA (Indeed Visual Concepts $\mathrm{GmbH})$.
\end{abstract}

\section{Introduction}

Precise geometrical reconstructions of neuronal architecture are essential for the investigation of neuronal function. Available commercial reconstruction software tools such as NEUROLUCIDA [1] provide manual tracing methods that are for complex dendritic trees overly tedious and lead to very subjective results. Moreover, by applying the reconstruction layer by layer, this method yields in stepwise structures. Thus, we applied our semi-automatic tracing method $[2,3]$, we are able to achieve a smoothly fitted geometry.

Compartmental models of neurons for computational purpose can be described using the so called cable equation. Herein the electrical properties of the intracellular fluid and of the neurons passive membrane are assigned to cylindrical compartments of the dendritic tree. The overall parameters Ra (axial resistance), $\mathrm{Rm}$ (membrane resistance) and $\mathrm{Cm}$ (membrane capacity) describe the specific electrical properties of the neuron, and as a first approximation they are assumed to be equal for each compartment. The time constant $\tau$, the electrotonic length $\lambda$, are values for the delay and decay of the voltage signal and were measured by biological experiments as well as the input resistance $R_{\mathrm{in}}$.

The state-of-the art visualization routines for modeling experiments in NEURON and GENESIS are not convenient for highly detailed reconstructions with 
about 80.000 compartments as in our case. Thus, for the purpose of rough modeling results we reduced the number of compartments. On the other hand, in order to avoid lack of precision, we transformed the visualization of voltage spread (e.g.) into color-coded time-stretched animations. As model we used the moth motoneuron MN5 at different stages of development, which changes from a slow motoneuron that is involved in larval crawling behaviour into a fast adult flight motoneuron. This metamorphosis is accompanied by changes in dendritic shape and electrophysiological properties of the neuron [4]. We addressed the question, how changes in dendritic structure can affect changes in functional behavior.

Highly detailed neuron models enhance the computational cost of neural modeling. Common routines to reduce the number of compartments with low loss of are optimized for neurons with less branching dendritic trees, and do not fulfill requrements of precise and fast modeling tasks with multi compartments as in our dimension.

\section{Materials and Methods}

\subsection{Imaging}

The imaging was performed on a Leica SP2 laser scanning confocal microscope by using a 40x oil immersion lens (NA1.25). The excitation maximum of the laser was at $543 \mathrm{~nm}$ and the detection range was $565-600 \mathrm{~nm}$. The voxel size was $(0.1 \mathrm{x} 0.1 \mathrm{x} 0.3) \mu \mathrm{m}^{3}[2]$. The complete $3 \mathrm{D}$ datasets were obtained sequentially at three different orientations as axial, sagittal and horizontal.

\subsection{Segmentation and Reconstruction of Dendritic Geometry}

The reconstruction starts with an initial coarse representation of the neuron, where all branching points (nodes) and their connectivity have to be defined by the user. With the tracing algorithm [2], a "snake" model was generated which consists of points ("snaxels") defined by their location, radii and edges that connect the nodes. The model is then fitted to the data, thus spanning the $3 \mathrm{~d}$ graph called skeleton, yielding precise shape and diameter of all dendrites.

\subsection{Reducing the Number of Compartments via Cylinder Fit}

The skeleton was transformed to cylindrical compartments into NEURON and GENESIS by means of our automated parsers [5]. This is useful because we can apply the cable equation. Herein the signal transmission is calculated by solving a simple differential equation for each time step in each cylinder segment. However, to enhance computational speed, several methods of cylinder fitting were investigated to reduce the number of compartments with a minimal loss of accuracy (Fig. 1). We have investigated three methods of reduction by evalating the same neuron model: first a systematic connection of a special amount of neighboring compartments, sececond the "radii-difference-method", and third a 
geometric optimization which constists of adding up the cylindrical lenghtes of a number of compartments, and subtituting the average of the radii for the resulting cylinder.

However, the optimal ratio of reduction in compartments to a minimal deviation in voltage transfer (compared to simulations with full number of compartments) resulted in our "radii-difference-method" (Fig. 1a). Here a cylinder is merged with its neighboring compartment, if the difference of their radii is less then a user defined range. The difference of the first and second cylinder (Fig. 1a, left) is within the range, so that the first cylinder was connected directly with the third one. The difference of the third and fourth cylinder was too large and cylinder four remains in the reconstruction. We have investigated to set the range to $0.1 \%, 0.5 \%, 1 \%, 5 \%$, and $10 \%$ of deviation.

The loss in computational accuracy was determined by computational simulations, and we compared the results with results of the not reduced model.

\subsection{Modeling}

In order to provide the model neuron with realistic passive membrane properties, three specific parameters ( $\mathrm{Ra}, \mathrm{Rm}$ and $\mathrm{Cm}$ ) were estimated from measurable values as time constant $(\tau)$, electronic length $(\lambda)$ and input resistance $\left(R_{\text {in }}\right)$. For this purpose we explored the parameter space of Ra, Rm and $\mathrm{Cm}$ by simulating voltage transfer inside the neuron, and compared the results for $R_{\text {in }}$ and $\lambda$ with the experimental measured values, see [6] for detailes.

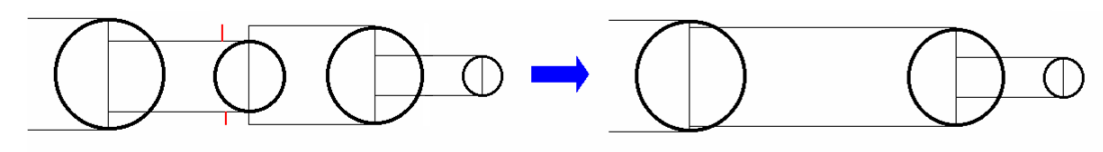

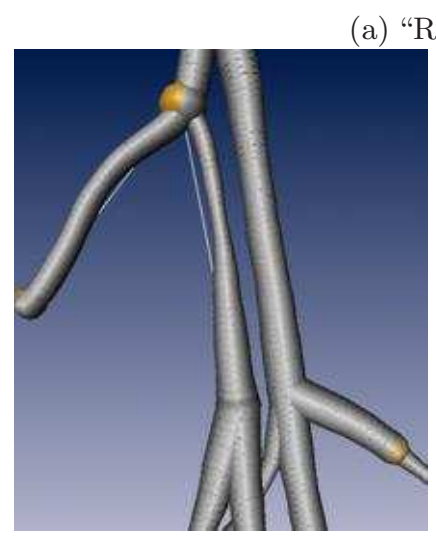

(b) Full Number of Compartments

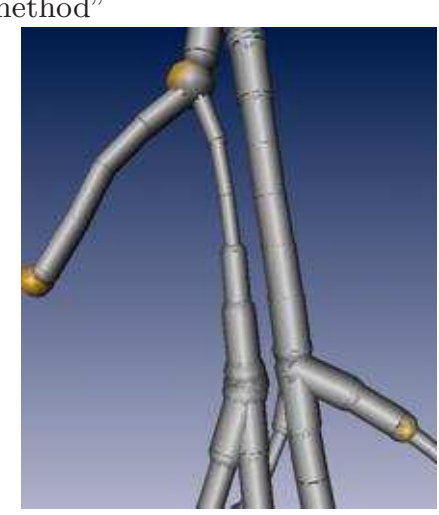

(c) Reduced Number of Compartments

Fig. 1. Results of the zylinder fit method 
We performed computational experiments, such as the voltage spread of an action potential (outward, Fig. 2a), and as postsynaptic signal transmission (inward, Fig. 2b), and addressed the following questions: a) Is an action potential capable to affect dendritic outgrowth by being back propagated into distal dendritic subtrees with only passive membrane properties? b) How can distal dendritic inputs affect the putative spike generation zone with passive membrane properties? c) Do depolarizing inputs to growth-cones affect the membrane potential of neighboring growth-cones?

\section{$3 \quad$ Results}

The joining of neighboring compartments with radius difference of less then $1 \%$ leads to a reduction of $58 \%$ of compartments with a voltage error of less then 0,18\%. Fig. 2 displays exemplary a specific time point of the spread of action potential. Equipotential regions in the dendritic tree are depicted by equivalent colors. The decrease of membrane potential from spike generation zone to distal dendritic subtrees is about $93 \%$. Vice versa, the voltage attenuation from distal dendritic tips to the root of the tree is about 99,8\%. Neighboring growth-cones affect each other with a voltage decay of $99,9 \%$.

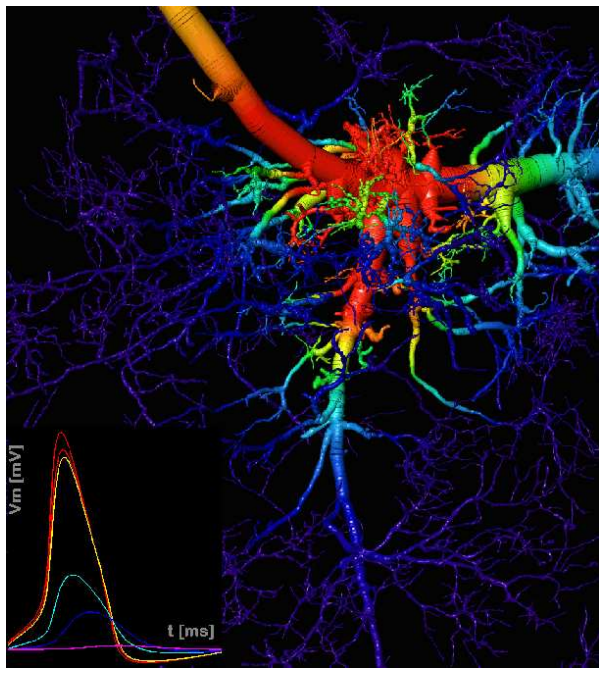

(a) Outward Spike

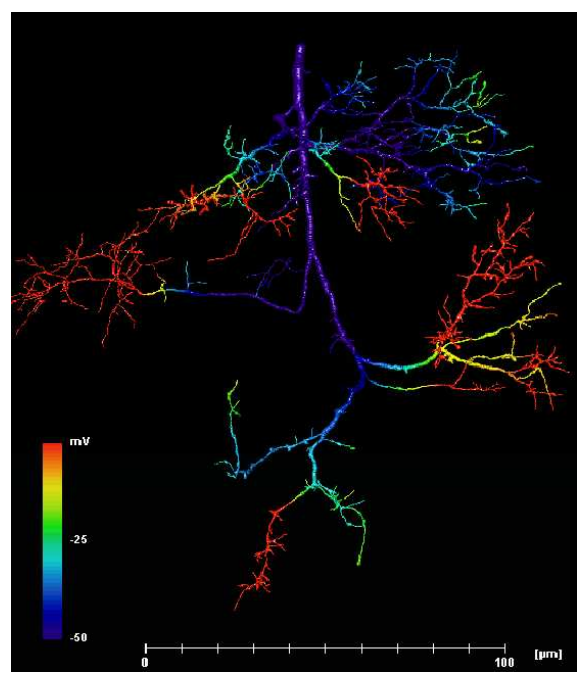

(b) Synaptic Integration

Fig. 2. Color-coded visualization of modeling results Both pictures display the part of the dendritic tree of the same reconstruction of themoth motoneuron MN5. Left: an outward spike was simulated. The voltage spread is depicted colorcoded throughout the dendritic tree. Inset: The time course of the voltage vor selected compartments. Red is close, blue is distal to the spike generation zone. Right: Inward signal integration. Tonic firing inputs to dendritic tips are integrated and processed to the dendritic root. The color bar is valid for both figures 


\section{Discussion}

We have used our semi-automatic reconstruction method and transformation routines to build highly detailed multi-compartmental models of the motoneuron MN5. To enhance computational speed we have successfully developed a method to reduce the number of cylindrical compartments within an acceptable error measure. Computational simulations were performed and imported in AMIRA for visualization.

In order to understand the processes in neurons with highly complex dendritic structure, it is essential to have also an intuitional impression of the modeling results. Thus, the voltage spreads, visualized in color-coded images reveal a most intuitively readable result that can be quantified by means of color bars (e.g). Moreover, our movie animations deliver intuitively the time course of signal transmission for each compartment throughout the dendritic tree, here depicted in terms of voltage-plots (Fig. 2a, inset).

Currently, our simulations focus on refining our model using recent experimental findings: A triangulated surface reconstruction of the neuron gives the possibility to extract a measure for synaptic localization from the second channel confocal data of immuno-labelled synapsin and to map this synaptotagmin distribution upon the skeleton [7]. We aim to include these data into our modeling experiments, and address the functional role of the structural changes for the observed lower excitability of the adult as compared to the larval motoneuron.

\section{References}

1. MicroBrightField, Inc, Williston, et al. Neurolucida.

2. Schmitt S, Evers J, Duch C, et al. New methods for the computer-assisted 3D reconstruction of neurons from confocal image stacks. NeuroImage. 2005;23:128398.

3. Evers J, Schmitt S, Scholz M, et al. New frontiers in functional neuroanatomy: Precise automatic reconstruction from confocal image stacks. J Neurophys. 2005;93:2331-42.

4. Duch C, Levine R. Remodeling of membrane properties and dendritic architecture accompanies the postembryonic conversion of a slow into a fast motoneuron. J Neurosci. 2001;20(18):6950-61.

5. Schönknecht S, Scholz M, Duch C, et al. Multi compartment model of developmental changes in dendritic shape during postembryonic motoneuron development. Proc Göttingen Neurobiol Conf. 2003.

6. Evers J, Maye A, Schönknecht S, et al. Are changing behavioural demands supported by postembryonic remodeling of neuronal structure? Proc Göttingen Neurobiol Conf. 2005;(55).

7. Evers J, Muench D, Duch C. Developmental relocation of presynaptic terminals along distinct types of dendritic filopodia. Developmental Biology. 2006;(297):241227. 